EXTENDED REPORT

\title{
Better efficacy of methotrexate given by intramuscular injection than orally in patients with rheumatoid arthritis
}

\author{
J Wegrzyn, P Adeleine, P Miossec
}

Ann Rheum Dis 2004;63:1232-1234. doi: 10.1136/ard.2003.011593

\begin{abstract}
See end of article for authors' affiliations

.....................

Correspondence to: Pr P Miossec, Clinical Immunology Unit, Departments of Immunology and Rheumatology, Hôpital Edouard Herriot, Lyon, 69437 Lyon Cedex 03 France; miossec@ univ-lyon $1 . f r$
\end{abstract}

Accepted

13 November 2003

\begin{abstract}
Objective: To compare the clinical efficacy of methotrexate and tolerance to the drug in patients with rheumatoid arthritis who were switched from intramuscular to oral administration because of a shortage of the intramuscular preparation.

Methods: 143 patients were switched from intramuscular to oral methotrexate. Of these, 47 were switched back to the intramuscular form. A multiple choice questionnaire was sent by mail to evaluate clinical and biological criteria of efficacy and tolerance.

Results: When methotrexate was first switched from intramuscular to oral administration, increased disease activity, exacerbation of morning pain and hand stiffness, duration of morning stiffness, increased joint pain, and increased joint swelling were observed. There was a greater frequency of gastrointestinal symptoms, but without a significant increase in liver abnormalities. When intramuscular methotrexate became available again, 47 of the 143 patients were switched back and were followed for at least three months. On average, disease manifestations were improved and side effects reduced by the switch.

Conclusions: Methotrexate given intramuscularly had improved clinical efficacy with fewer side effects than given orally. Intramuscular methotrexate administration should be considered when rheumatoid arthritis remains active in spite of high dose oral methotrexate.
\end{abstract}

M ethotrexate is now the most commonly used slow acting antirheumatic drug for rheumatoid arthritis. ${ }^{12}$ In contrast to anticancer treatment, very low doses are given weekly ( 7.5 to $25 \mathrm{mg} /$ week) by the oral, intramuscular, or subcutaneous route. ${ }^{3}$ Intramuscular administration is desirable because there is better absorption than by the oral route, the peak concentrations are similar to the intravenous (IV) route, and there is slower drug absorption and more prolonged exposure to the drug than with IV administration. ${ }^{4}$ Conversely, oral methotrexate may be associated with reduced gastrointestinal tolerance. ${ }^{5}$ Previous studies have shown better efficacy of intramuscular than oral methotrexate, based on pharmacokinetic indices of absorption and bioavailability, ${ }^{4}$ but to our knowledge clinical variables were evaluated in only one study. ${ }^{6}$ This latter study showed better efficacy with intramuscular methotrexate and a low incidence of adverse events.

There is at present no clear consensus on the route of choice for methotrexate administration, which differs between countries according to the prevailing medico-social systems and legislation. In the USA, methotrexate is mostly given orally, whereas in countries such as France, intramuscular administration is more common, although not yet legally approved.

From June to September 2001, the injectable form of methotrexate was out of stock in several European countries. Patients treated with intramuscular methotrexate thus had to be switched to the oral form. This transient shortage of the product gave us an opportunity to compare intramuscular and oral administration for efficacy and tolerance to the drug. A group of 143 patients switched from intramuscular to oral methotrexate was analysed in a retrospective and longitudinal study. When the supply problem was solved a few months later, some could be switch back to the intramuscular form, allowing a crossover comparison.

\section{METHODS}

We selected 213 patients from a computer database of the patients followed at the rheumatology unit of the Hôpital
Edouard Herriot, Lyon, France. To be included, patients had to have rheumatoid arthritis, to be on a stable dose of intramuscular methotrexate as of June 2001, and to have been switched to oral methotrexate from June to September 2001 only because of a shortage of the intramuscular form. Methotrexate dosage was kept identical. We excluded patients already on the oral preparation, those stopping methotrexate because of side effects or lack of efficacy, those for whom intramuscular methotrexate was already insufficient, and those refusing injections. Patients already on folic acid were maintained on the same dose.

A multiple choice questionnaire was created, first tested on 10 eligible patients and sent by mail on January 2002. The aim was to evaluate the following:

- Il clinical criteria (joint pain, morning pain, joint swelling, hand morning stiffness, duration of morning joint swelling, consumption of analgesic drugs and its duration, eye and mouth dryness, nausea after taking methotrexate);

- one biological criterion (transaminase levels).

In those in whom intramuscular methotrexate was reintroduced in September 2001 the same criteria were evaluated three months after the switch from the oral to the intramuscular route. For each question, possible answers were an increase, a decrease, or no change.

\section{Statistical analysis}

Analysis of the results was carried out using the SPSS program (SPSS Inc, Chicago Illinois, USA). A descriptive statistical analysis was undertaken using contingency tables for each criterion. In the group of patients with a crossover comparison, tables with a two dimension distribution were used to analyse the effects of changing from intramuscular to oral methotrexate and then from oral to intramuscular. The $\chi^{2}$ test was used to evaluate changes for each criterion. A probability $(p)$ value of ?0.05 was considered as significant. 


\section{RESULTS}

\section{Analysis of the study population}

The response rate to the mail questionnaire was $76 \%$ (162 of 213). Of the 51 patients who did not reply, five had died, 36 were unconcerned, and 10 were lost of follow up. Because of geographical heterogeneity in the shortage of parenteral methotrexate, 19 patients who had continued taking intramuscular methotrexate during this period were excluded. The final analysis involved 143 patients with rheumatoid arthritis (129 women and 14 men), mean age 65 years (range 21 to 86 ) (table 1). From this patient group, 47 were switched back to intramuscular methotrexate in September 2001. There was no significant difference at the time of the intramuscular to oral switch between the patients who were switched back or not from oral to intramuscular administration with respect to the variables listed in table 1.

\section{Effect of switch from intramuscular to oral methotrexate}

When methotrexate was first switched from intramuscular to oral in these 143 patients with rheumatoid arthritis, most of them experienced increased disease activity. These changes are shown in table 1 . Seventy $(49 \%)$ had exacerbation of morning pain $(\mathrm{p}<0.001), 92(64 \%)$ had increase morning hand stiffness $(p<0.001), 89(63 \%)$ had increased duration of morning stiffness $(\mathrm{p}<0.001), 102(71 \%)$ had more joint pain $(\mathrm{p}<0.001)$, and $85(59 \%)$ had increased joint swelling $(\mathrm{p}<0.001)$. In response to this increased disease activity, 94 $(66 \%)$ increased their consumption of analgesics, which were taken for a longer duration $(\mathrm{p}<0.001)$. Conversely, $82(57 \%)$ of the patients found no change in eye dryness and $71(50 \%)$ found no difference in mouth dryness. Regarding tolerance, 69 patients $(48 \%)$ experienced nausea after taking oral methotrexate $(\mathrm{p}<0.001)$. This could not be explained by differences in the use of folic acid. With respect to liver toxicity, an increase in transaminases was found in only $16 \%$ of the patients (NS). Overall, the outcome of the switch from intramuscular to oral administration was a lower efficacy for treating arthritis symptoms but no effect on the sicca syndrome. An increased frequency of gastrointestinal events with the oral preparation was not associated with an increased frequency of liver abnormalities.

\section{Effect of oral to intramuscular methotrexate switch} When intramuscular methotrexate again became available, 47 of the 143 patients were switched back and could be followed for at least three months. Most of these 47 patients experienced decreased disease activity within three months of resumption of intramuscular treatment. Table 2 shows the changes when intramuscular methotrexate was changed to oral, and when intramuscular treatment was reintroduced. With respect to efficacy, the most common pattern was an increased intensity of symptoms when methotrexate was used orally, followed by a decrease in intensity when it was switched back to intramuscular. In the group changing to oral and then back to intramuscular treatment, an exacerbation followed by an improvement occurred in the following indices: morning pain (20 patients $(42 \%) ; \mathrm{p}<0.001)$, morning hand stiffness (23 patients $(49 \%) ; \mathrm{p}<0.001)$, duration of morning stiffness ( 28 patients $(60 \%) ; \mathrm{p}<0.001$ ), joint pain (33 patients $(70 \%) ; \mathrm{p}<0.001)$, joint swelling ( 19 patients $(40 \%) ; p<0.001)$, increased consumption of analgesic drugs (30 patients $(63 \%) ; p<0.001)$, and increased duration of analgesic use (31 patients $(65 \%) ; \mathrm{p}<0.001)$. There was no change in eye dryness in 22 patients $(47 \%)$ or in oral dryness in 19 patients $(40 \%)$. Regarding tolerance, 19 patients $(40 \%)$ still had nausea even with intramuscular methotrexate. This could not be explained by a difference in folic acid use. With respect to liver toxicity, there was no change in transaminase values in 23 patients $(49 \%)$, while in $10(21 \%)$ an increase on oral treatment was followed by a decrease on intramuscular treatment. However, 11 patients $(23 \%)$ did not notice any change in the frequency or intensity of symptoms when oral methotrexate was switched back to intramuscular administration. Overall, switching back to intramuscular administration resulted in more effective control of disease manifestations, while at the same time the incidence of side effects was reduced. However, an increased treatment burden was the main reason given by 89 of the 96 patients who did not switch back to intramuscular administration.

\section{DISCUSSION}

Several studies have compared the various modes of methotrexate administration. ${ }^{45}$ Oral methotrexate is rapidly but incompletely absorbed (approximately 15\% less than intramuscular), although with large interindividual variability. ${ }^{9}$ For doses above $25 \mathrm{mg} / \mathrm{day}$, oral administration is associated with lower bioavailability because of absorption saturation. ${ }^{10}$ Conversely, intramuscular administration results in rapid and complete absorption and higher serum concentrations. ${ }^{7}$

Although these pharmacological studies have shown that intramuscular methotrexate should work better, few studies have examined the clinical implications of such a switch. ${ }^{60-12}$ As revealed here, a relapse in the clinical symptoms of rheumatoid arthritis may be observed after switching from intramuscular to oral administration..$^{10} \mathrm{~A}$ recent open study comparing oral with intramuscular methotrexate also showed increased efficacy with fewer adverse events with intramuscular administration. ${ }^{6}$ Such findings imply that a switch to the intramuscular route could be useful in patients not responding adequately to the oral drug. ${ }^{11}$

To evaluate the effect of switching from intramuscular to oral administration, we took advantage of the fact that

Table 1 Effects of a switch from intramuscular to oral methotrexate in 143 patients

\begin{tabular}{lllll}
\hline & Increase & No change & Decrease & Not answered \\
\hline Morning pain & $70(49)$ & $59(41)$ & 0 & $14(10)$ \\
Hand morning stiffness & $92(64)$ & $49(34)$ & 0 & $2(2)$ \\
Duration of morning joint stiffness & $89(63)$ & $49(34)$ & 0 & $5(3)$ \\
Joint pain & $102(71)$ & $41(29)$ & 0 & 0 \\
Joint swelling & $85(59)$ & $49(34)$ & 0 & $9(7)$ \\
Consumption of analgesics & $94(66)$ & $45(31)$ & 0 & $4(3)$ \\
Duration of analgesic consumption & $94(66)$ & $45(31)$ & 0 & $4(3)$ \\
Eye dryness & $19(14)$ & $82(57)$ & 0 & $42(29)$ \\
Mouth dryness & $27(19)$ & $71(50)$ & 0 & $45(31)$ \\
Nausea after taking methotrexate & $69(48)$ & $32(22)$ & 0 & $42(29)$ \\
Transaminase levels & $23(16)$ & $89(62)$ & 1 & $30(22)$ \\
\hline Results are expressed as $\mathrm{n}(\%)$. & & & \\
\hline
\end{tabular}




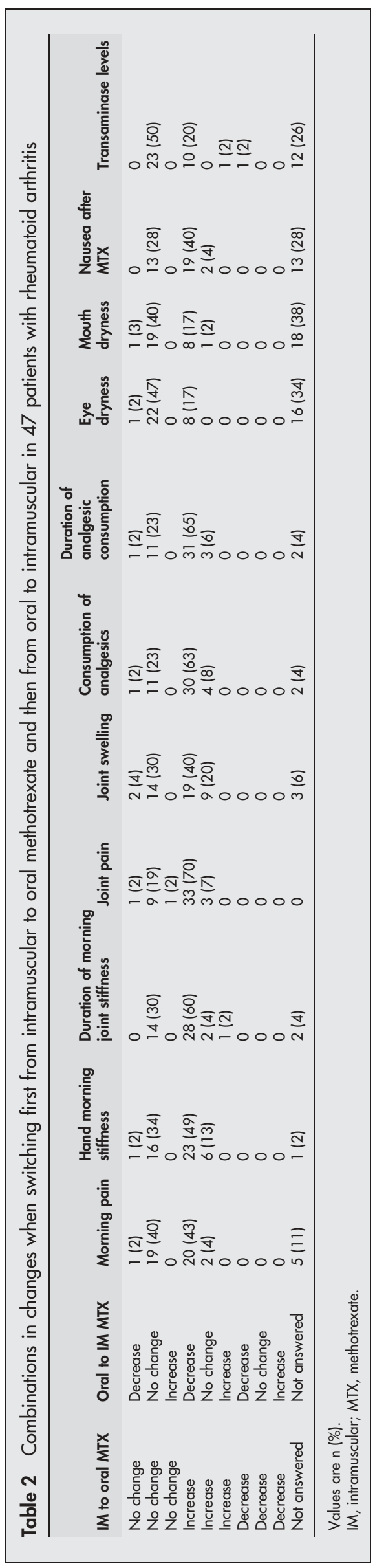

between June and September 2001 intramuscular methotrexate was out of stock in France. In our study of 143 patients with rheumatoid arthritis initially treated with intramuscular methotrexate, each patient remained their own control. All the clinical symptoms of rheumatoid arthritis were exacerbated by the change. As an internal control, oral and eye dryness did not change, neither did transaminase values.

As the hiatus in supply of intramuscular methotrexate was brief, 47 patients could be switched back to the intramuscular preparation, allowing a crossover analysis. When comparing the two modes of administration, most patients showed an increase in disease activity on the oral preparation, followed by a decrease on the intramuscular preparation. With respect to toxicity, although there was no significant overall change in liver transaminases, the values increased in nearly a quarter of the patients when they were on oral methotrexate and decreased again when they were re-established on intramuscular methotrexate, suggesting reduced liver toxicity with the intramuscular preparation. ${ }^{5}{ }^{12}$ A reduction in side effects permits higher doses to be used, leading in turn to better efficacy. However, an increase in the treatment burden and cost is associated with intramuscular methotrexate.

\section{Conclusions}

Our observations are in line with other studies ${ }^{70-12}$ and should be taken into account in approving intramuscular methotrexate for the treatment of rheumatoid arthritis. It remains to be shown whether intramuscular administration increases the efficacy of the drug in patients unresponsive to oral administration. Finally, the mode of administration should be considered when comparing its effect with cytokine inhibitors, particularly when rheumatoid disease is active despite high dose oral methotrexate.

\section{Authors' affiliations}

J Wegrzyn, P Miossec, Departments of Immunology and Rheumatology, Hôpital Edouard Herriot, Lyon, France

P Adeleine, Departments of Epidemiology and Medical Information, Hospices Civils de Lyon

\section{REFERENCES}

1 Lentendre PW, DeJong DJ, Miller DR. The use of methotrexate in rheumatoid arthritis. Drug Intell Clin Pharm 1985; 19:349-58.

2 Haraoui B, Pelletier JP, Cloutier JM, Faure MP, Martel-Pelletier J. Synovial membrane histology and immunopathology in rheumatoid arthritis and osteoarthritis: in vivo effects of antirheumatic drugs. Arthritis Rheum 1991;34:153-63.

3 Arthur V, Jubb R, Homer D. A study of parenteral use of methotrexate in rheumatic conditions. J Clin Nurs 2002;11:256-63.

4 Freeman-Narrod M, Gerstley BJ, Engstrom PF. Comparison of serum concentrations of methotrexate after various routes of administration. Cancer 1975;36:1619-24.

5 Kremer JM, Lee JK. The safety and efficacy of the use of methotrexate in long term therapy for rheumatoid arthritis. Arthritis Rheum 1986;29:822-31

6 Bingham SJ, Buch H, Lindsay S, Pollard A, White J, Emery P. Parenteral methotrexate should be given before biological therapy. Rheumatology 2003:42:1009-10

7 Hamilton RA, Kremer JM. Why intramuscular methotrexate may be more efficacious than oral dosing in patients with rheumatoid arthritis. BrJ Rheumatol 1997;36:86-90.

8 Campbell MA, Perrier DG, Dorr RT, Alberts DS, Faley PR. Methotrexate: bioavailability and pharmacokinetics. Cancer Treat Rep 1985;69:833-8.

9 Furst DE. Practical clinical pharmacology and drug interactions of low-dose methotrexate therapy in rheumatoid arthritis. Br J Rheumatol 1995;34(suppl 2):20-5.

10 Rozin A, Schapira D, Balbbir-Gurman A, et al. Relapse of rheumatoid arthritis after substitution of oral for parenteral administration of methotrexate. Ann Rheum Dis 2002;61:756-7.

11 Osman A, Mulherin D. Is parenteral methotrexate worth trying? Ann Rheum Dis $2001 ; 60: 432$

12 Burbage G, Gupta R, Lim K. Intramuscular methotrexate in inflammatory rheumatic disease. Ann Rheum Dis 2001;60:1156. 